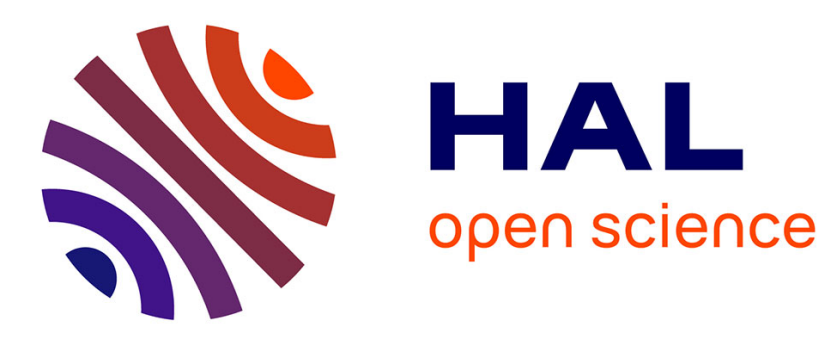

\title{
Approximation of the Vehicle Stability Domain using Interval Analysis
}

\author{
Hoda Dandach, Jérôme de Miras, Ali Charara
}

\section{To cite this version:}

Hoda Dandach, Jérôme de Miras, Ali Charara. Approximation of the Vehicle Stability Domain using Interval Analysis. 16th IEEE International Annual Conference on Intelligent Transportation Systems (ITSC 2013), Oct 2013, The Hague, Netherlands. pp.91-98. hal-00923323

\section{HAL Id: hal-00923323 \\ https://hal.science/hal-00923323}

Submitted on 2 Jan 2014

HAL is a multi-disciplinary open access archive for the deposit and dissemination of scientific research documents, whether they are published or not. The documents may come from teaching and research institutions in France or abroad, or from public or private research centers.
L'archive ouverte pluridisciplinaire HAL, est destinée au dépôt et à la diffusion de documents scientifiques de niveau recherche, publiés ou non, émanant des établissements d'enseignement et de recherche français ou étrangers, des laboratoires publics ou privés. 


\title{
Approximation of the Vehicle Stability Domain using Interval Analysis*
}

\author{
Hoda Dandach $^{1}$ and Jérôme De Miras ${ }^{1}$ and Ali Charara ${ }^{1}$
}

\begin{abstract}
This paper proposes a new approach to compute and predefine a state space where the vehicle is stable, using interval analysis. We call this state space the vehicle stability domain. The vehicle state $p$ is composed of the longitudinal and lateral accelerations at the center of gravity. The vehicle stability is described using risk indicators as the rollover index $L T R$ of the vehicle, the longitudinal slip ratio, and the side slip angle of each wheel. These three elements form the vector y. We compute the set of accelerations that correspond to acceptable values of $y$. We use interval analysis to give inner and outer approximations of this set with a maximal guaranteed accuracy. In order to illustrate the principle and efficiency of the approach, a validation with simulated data is provided.
\end{abstract}

\section{INTRODUCTION}

Road safety is a major issue affecting the public health of all nations. A lot of research focus on how to increase it. The main purpose is to keep the vehicle in a stable state where over steering, under steering, rollover, or sliding, etc. are avoided, and its responses are controlled, especially in critical situations. A domain of this stable state could be predefined off-line using risk indicators and vehicle dynamics. In this paper, we are interested in computing this domain in order to have it as a useful information to any other system of command or state observation. It will be as a prerequisite of the classical observation and control methods.

Classically, measurements and state estimation are ways and methods to know the actual vehicle state. Vehicles are equipped with proprioceptive sensors as gyrometers and accelerometers. Gyrometers measure the angular motion of the vehicle and its wheels, while accelerometers measure linear motion and gravity. The measurements of these elements of the vehicle state are used in control loops dedicated to handle its behavior [11]. Other state elements as the vertical forces created between each wheel and the road, the friction coefficient, the side slip angle at the vehicle center of gravity, etc. are very expensive to measure. We estimate these variables using the other inexpensive measurements [4], [6]. Estimation of these variables and parameters related to the vehicle dynamics can provide the driver a warning of a possible loss of vehicle control. Anticipating these elements may prevent such situations [7]. Exteroceptive sensors as a GPS receiver, a camera, a lidar, etc. are used to help the driver (and eventually the autonomous vehicles) to locate his vehicle, detect and classify the obstacles and the pedestrians,

\footnotetext{
*This work is supported by the french regional project ACADIE and the European Fund of Regional Development FEDER.

${ }^{1}$ H. Dandach, J. De Miras and A. Charara are with Heudiasyc, UMR CNRS 7253, Université de Technologie de Compiègne, Centre de Recherches de Royallieu, BP 20529, 60205 Compiègne Cedex, France. hoda.dandach@utc.fr, demiras@hds.utc.fr, ali.chararaehds.utc.fr
}

and generate safe trajectories [16], [13]. These tasks, as well as the state estimation, are based on the noisy sensors measurements. Bayesian filters, such as the Kalman filter (KF) in its three forms (linear, non linear and unscented), are one of the most popular and preferred solution for these issues, mainly because of their simplicity and low memory requirements [1]. These probabilistic filters generate a point state estimation.

Introducing the interval framework, interval state estimation seems more guaranteed than a point state estimation especially when the uncertainties on the system dynamics and the measurements are bounded. Interval framework deals with the problem of the noisy measurements. It does not assume a unimodal Gaussian state and measurement distributions as in Kalman filters. In [3], the box particle filter approach (BPF) combining the Bayesian framework with interval methods succeeds in estimating the roll angle, the lateral acceleration, and the lateral load transfer of the vehicle. BPF gives a guaranteed estimation of the state vector. The box encountering the estimation is guaranteed to encounter the real value of the estimated variable as well. In [5], a confidence domain of position is given by solving a constraint satisfaction problem using interval analysis.

State estimation, using either probabilistic or interval based technics, is a basic pre-build step for command laws of systems. On the other hand, every system has a stable evolution zone, if it exists, or an unstable one. Predefining a space where the state could vary while the system is staying within its stable evolution zone seems interesting for the following estimation and command laws building steps. Using interval analysis, an inner approximation of this predefined stable state space reduces the variables explored domain to a smaller one where, having the system evolution model, it is guaranteed to stay stable against risks. The resultant domain will be called stability domain. In this paper, we are concerned about computing an approximation of the vehicle stability domain against the risks of rollover, under or over steering and slipping on the road.

This paper is organized as follows. After an overview of the vehicle dynamics, we propose the limits of the vehicle stability in section II. Interval analysis and set inversion problem are introduced in section III. The proposed method for computing the stability domain using an interval solver and the simulated validation are presented with details in section IV. Finally, the conclusion of this work and the perspectives are presented in section V. 


\section{VEHICLE DYNAMICS}

A realistic modelling of the vehicle dynamics is essential to understand its behavior. The more the model is simple and reliable, the better it is for computing and control. In the following, a brief description of the four-wheel vehicle dynamics used in this paper is presented.

\section{A. Vehicle Model}

Illustrated in the Fig. 1 and Fig. 2, some variables and parameters describing the state of the vehicle are [10],[14],[17]:

- $F_{x i j}, F_{y i j}$ and $F_{z i j}$ : Longitudinal, lateral and vertical forces $(N)$ respectively, generated through contact of the wheel $i j$ with the road. Index $i \in\{1,2\}$ represents the front and the rear wheels and index $j \in\{1,2\}$ represents the left and the right wheels.

- $\beta$ and $\alpha_{i j}$ : Side slip angle at the vehicle center of gravity (CoG) and the side slip angle of the wheel $i j$ (named as well lateral slip) respectively $(\mathrm{rad})$.

- $V_{g}$ : Velocity vector at the CoG $(\mathrm{m} / \mathrm{s})$.

- $V_{x}$ and $V_{y}$ : Longitudinal and lateral velocities at the CoG respectively $(\mathrm{m} / \mathrm{s})$.

- $a_{x}$ and $a_{y}$ : Longitudinal and lateral accelerations at the CoG $\left(m / s^{2}\right)$.

- $\dot{\psi}$ : Yaw rate $(\mathrm{rad} / \mathrm{s})$.

- $\theta$ : Roll angle (rad).

- $\delta$ : Steering angle $(\mathrm{rad})$.

- $m$ : Total vehicle mass $(k g)$.

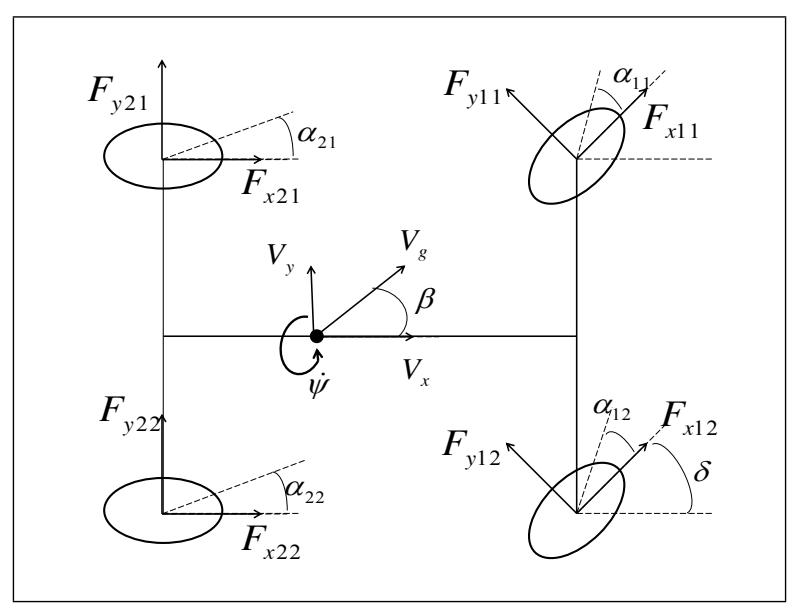

Fig. 1. Four-wheel vehicle model

The vertical forces are mainly generated by the weight of the vehicle. Longitudinal and lateral accelerations affect the values of these forces. Due to the vehicle inertia, a positive longitudinal acceleration $a_{x}$ creates a pitch motion which relieves the front axle and loads the rear axle. As well, during cornering, the roll torque created by the lateral acceleration $a_{y}$ increases the load on the outside and decreases it on the inside of the vehicle. Equations (1) illustrate the relation between vertical forces of each wheel, the longitudinal and the lateral accelerations [10].

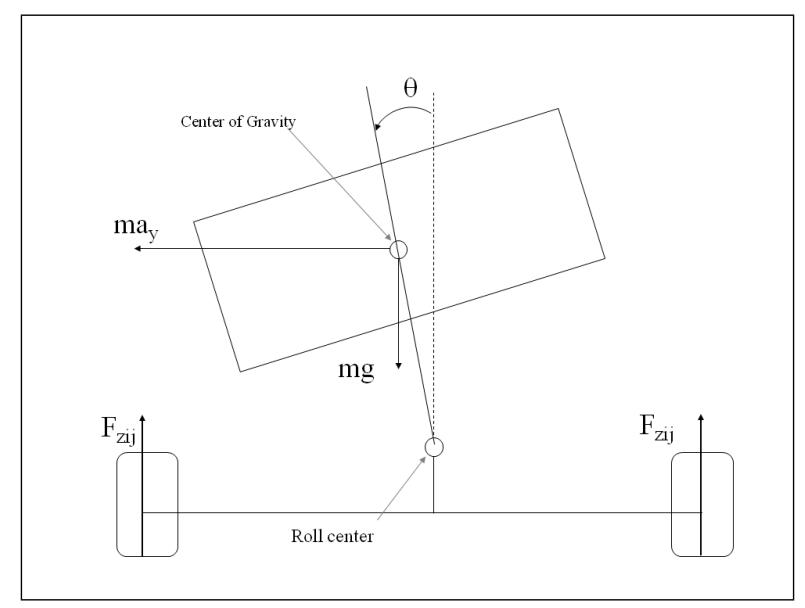

Fig. 2. Roll plan model

$$
\left\{\begin{array}{l}
F_{z 11}=m\left(\frac{l_{r} g}{l}-\frac{h a_{x}}{l}\right)\left(0.5-\frac{h a_{y}}{b_{f} g}\right) \\
F_{z 12}=m\left(\frac{l_{r} g}{l}-\frac{h a_{x}}{l}\right)\left(0.5+\frac{h a_{y}}{b_{f} g}\right) \\
F_{z 21}=m\left(\frac{l_{f} g}{l}+\frac{h a_{x}}{l}\right)\left(0.5-\frac{h a_{y}}{b_{r} g}\right) \\
F_{z 22}=m\left(\frac{l_{f} g}{l}+\frac{h a_{x}}{l}\right)\left(0.5+\frac{h a_{y}}{b_{r} g}\right)
\end{array}\right.
$$

where $h$ is the height of the vehicle center of gravity in meters, $b_{f}$ and $b_{r}$ are the front and the rear vehicle's track respectively in meters, $l_{f}$ and $l_{r}$ are the distance from the center of gravity to the front and the rear axles respectively in meters, $l=l_{f}+l_{r}$ is the wheelbase in meters, $m$ is the total vehicle mass in kilograms, and $g$ is the gravitational acceleration in meters per square second.

By applying Newton's second law to the vehicle mass longitudinally and laterally around the vertical axis passing through its center of gravity, we obtain the motion equations (2) describing the relationship between $a_{x}, a_{y}, F_{x i j}, F_{y i j}$ for all $i \in\{1,2\}$ and $j \in\{1,2\}$.

$$
\left\{\begin{aligned}
a_{x}= & \frac{1}{m}\left(-\left(F_{y 11}+F_{y 12}\right) \sin \delta+\left(F_{x 11}+F_{x 12}\right) \cos \delta\right. \\
& \left.+F_{x 21}+F_{x 22}\right) \\
a_{y}= & \frac{1}{m}\left(\left(F_{y 11}+F_{y 12}\right) \cos \delta+\left(F_{x 11}+F_{x 12}\right) \sin \delta\right. \\
& \left.+F_{y 21}+F_{y 22}\right)
\end{aligned}\right.
$$

A mathematical formula known as "Magic formula" proposed by Pacejka [14], given in (3), shows the basic expressions of the longitudinal and lateral forces. These forces are functions of the longitudinal slip ratio $\sigma_{i j}$ and the wheel side slip angle $\alpha_{i j}$ of each wheel $i j, i \in\{1,2\}$ and $j \in\{1,2\}$. For simplicity, $F_{x}, F_{y}, \sigma$ and $\alpha$ replace in these equations $F_{x i j}, F_{y i j}, \sigma_{i j}$ and $\alpha_{i j}$ respectively. The Fig. 3 and Fig. 4 show how $F_{x}$ and $F_{y}$ vary with $\sigma$ and $\alpha$.

$\left\{\begin{array}{l}F_{x}=D_{x} \sin \left(C_{x} \arctan \left(B_{x} \sigma-E_{x}\left(B_{x} \sigma-\arctan \left(B_{x} \sigma\right)\right)\right)\right) \\ F_{y}=D_{y} \sin \left(C_{y} \arctan \left(B_{y} \alpha-E_{y}\left(B_{y} \alpha-\arctan \left(B_{y} \alpha\right)\right)\right)\right)\end{array}\right.$

where $B$ is the stiffness factor, $D=\mu F_{z}$ is the peak factor ( $\mu$ is the friction coefficient). The other parameters $C, E$ and 
$\mu$ [6] can be estimated or predetermined using regression techniques.

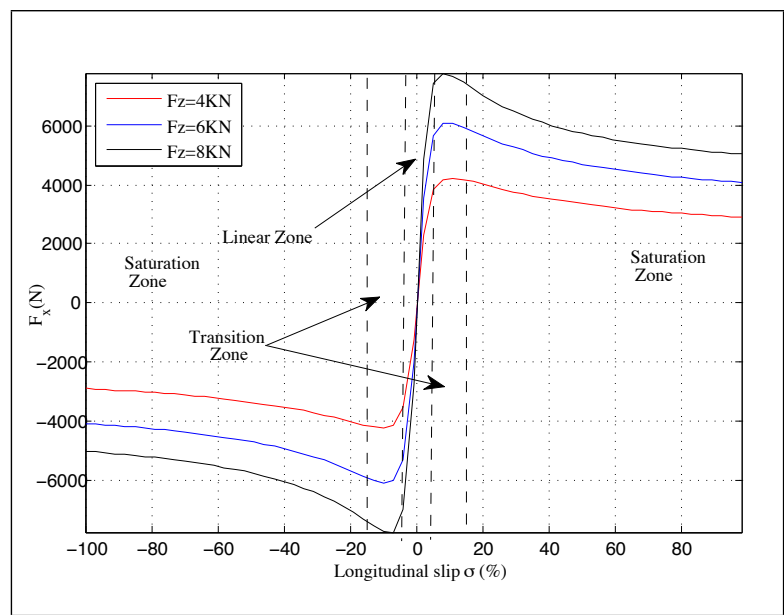

Fig. 3. Pacejkas Model: longitudinal force $F_{x}$ vs. longitudinal slip ratio $\sigma$ for different loads $F_{z}$

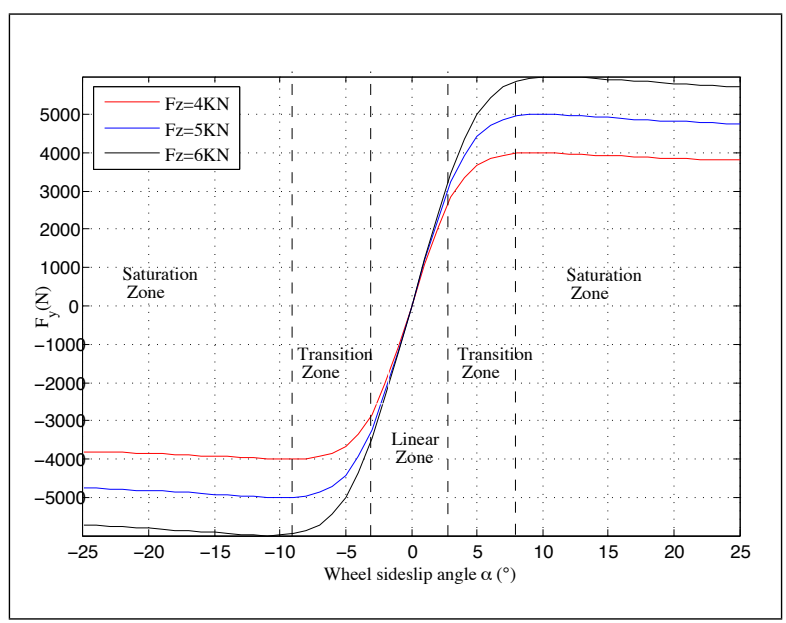

Fig. 4. Pacejkas Model: lateral force $F_{y}$ vs. wheel side slip angle $\alpha$ for different loads $F_{z}$

\section{B. Stability domain}

Vehicle rollover is one of the most life-threatening factors in car accidents. Entering a curve with an excessive speed may induce it. An important challenge is to design an index describing the vehicle's dynamic roll behavior [2], [15], [17]. The lateral transfer ratio $L T R$, given in (4), is a reliable rollover prediction index. It is the ratio of the difference between the sum of the left wheel vertical forces $\left(F_{z 11}\right.$ and $\left.F_{z 21}\right)$ and the right wheel ones $\left(F_{z 12}\right.$ and $\left.F_{z 22}\right)$ to the sum of all the wheel vertical forces. The value of $L T R$ varies from -1 (lift-off of the left wheels), 0 (no load transfer) and 1 (lift-off of the right wheels). When the absolute value of $L T R$ exceeds 0.8 , we consider that the vehicle is in danger and out of its stability domain.

$$
L T R=\frac{\left(F_{z 11}+F_{z 21}\right)-\left(F_{z 12}+F_{z 22}\right)}{\sum_{i=1}^{2} \sum_{j=1}^{2} F_{z i j}}
$$

As it can be easily seen in Fig. 3 and Fig. 4, three different tire behavior zones can be distinguished: linear, transition and saturation zones. In Fig. 3, during driving, a positive $\sigma$ induces a positive longitudinal force $F_{x}$. While driving on slippery roads, $\sigma$ may attain very large values in the saturation mode. While braking, $\sigma=-1$ corresponds to wheel lock.

The lateral force $F_{y}$ increases linearly with the side slip angle in the linear zone. In the saturation zone, the wheel slips. For example, if the front wheels saturate first, the vehicle under steers. If the rear wheels saturate first, it over steers. Controlling the vehicle in these situations requires professionalism which is not a common quality among normal drivers. In consequence, these behaviors become particularly dangerous.

Thus, avoiding transition zones and eventually saturation zones is crucial for the vehicle's handling and stability performance. We consider that above a longitudinal slip ratio equal to $15 \%$ and a sideslip angle $\alpha$ equal to $6^{\circ}$ (which is equal to $0.1 \mathrm{rad}$ ), $F_{x}$ and $F_{y}$ enter in their respective transition zones and later in there respective saturation zones. We need to avoid such a behavior of vehicle forces. Below these values, a normal driver can easily control its vehicle especially with active safety systems as ABS, EPS, etc. and bring it back to stability in case of slipping, overteering or other critical situations.

In conclusion, in this paper, we consider that the vehicle stability domain is defined in the zone where the absolute value of the rollover index $L T R$ is below 0.8 , the longitudinal slip ratio is up to $15 \%$, and the wheel sideslip angle is up to $6^{\circ}$ as summarized in Table. I.

TABLE I

Vehicle STABILITY Domain

\begin{tabular}{|c||c|}
\hline Parameter & Stability Domain \\
\hline Rollover index $L T R$ & {$[-0.8,0.8]$} \\
\hline Longitudinal slip ratio $\sigma(\%)$ & {$[-15,15]$} \\
\hline Wheel side slip angle $\alpha\left(^{\circ}\right)$ & {$[-6,6]$} \\
\hline
\end{tabular}

\section{INTERVAL ANALYSIS AND SET INVERSION}

\section{A. Interval Analysis}

Interval analysis [8] is a guaranteed numerical method used for approximating sets. The guarantee is to obtain at least an outer approximation of the set of interest with the desired precision. Real numbers are enclosed in intervals, and real vectors in boxes. An interval $[x]=[\underline{x}, \bar{x}]$ is a connected subset of $\mathbb{R}$, where $\underline{x}$ and $\bar{x}$ are respectively the lower and upper bounds of $[x]$ as illustrated in (5):

$$
[\underline{x}, \bar{x}]=\{x \in \mathbb{R} \mid \underline{x} \leq x \leq \bar{x}\}
$$

We denote by $\mathbb{I R}$ the set of all such intervals (6):

$$
\mathbb{I} \mathbb{R}=\{[\underline{x}, \bar{x}] \mid \underline{x}, \bar{x} \in \mathbb{R} \text { and } \underline{x} \leq \bar{x}\}
$$


An interval vector or box (in bold) $[\mathbf{x}]$ is a subset of $\mathbb{R}^{n}$ defined as the cartesian product of $n$ closed intervals as in (7):

$$
[\mathbf{x}]=\left[x_{1}\right] \times\left[x_{2}\right] \times \cdots \times\left[x_{n}\right]
$$

As well, it is denoted: $[\mathbf{x}]=\left(\left[x_{1}\right], \ldots,\left[x_{n}\right]\right)^{T} . \mathbb{I}^{n}$ is the set of all $n$-dimensional boxes.

Interval arithmetic [12] defines operations on intervals. The four basic operations on reals,,$+- \times$ and $\div$ can be extended to operate on intervals. Let $\diamond$ be any of these operators. $[x] \diamond[y]$ returns the smallest interval containing the result as illustrated in (8):

$$
[x] \diamond[y]=\{x \diamond y \in \mathbb{R} \mid x \in[x], y \in[y]\}
$$

For example, $[0,4]-[1,3]=[-3,3]$. Similarly, we extend other elementary functions such as exp, sin, cos, tan, sqr, $\cdots$ to intervals. Consider $f: \mathbb{R} \rightarrow \mathbb{R}$ one of these functions. Its extension on intervals is as in (9):

$$
[f]([x])=\{f(x) \mid x \in \mathbb{R}\}
$$

For example, $[s q r]([-1,3])=[0,9]$.

Interval analysis [8] is based on the set-theory. In consequence, sets operations can be as well applied on intervals. The result is always the smallest closed interval containing the answer. For instance, the intersection of two intervals is given in (10):

$$
[x] \cap[y]=\{z \in \mathbb{R} \mid z \in[x] \text { and } z \in[y]\}
$$

Consider a function $f: \mathbb{R}^{n} \rightarrow \mathbb{R}^{m}$. The image of a box $[\mathbf{x}]$ with $f$, denoted by $f([\mathbf{x}])$, is not necessary a box and could have any shape. To solve this problem, we define $[f]$ : $\mathbb{I}^{n} \rightarrow \mathbb{\mathbb { R } ^ { m }}$ as an inclusion function for $f$ if it returns a box guaranteed to contain $f([\mathbf{x}])$ as in (11):

$$
\forall[\mathbf{x}] \in \mathbb{R}^{n}, f([\mathbf{x}]) \subset[f]([\mathbf{x}])
$$

$[f]$ is called minimal if it returns the smallest box containing the image set $f([\mathbf{x}])$. There exist many methods to construct an inclusion function for $f$. One of these functions is called the natural inclusion function. Consider the function $f$ as in (12):

$$
\begin{aligned}
f: & \mathbb{R}^{n} \rightarrow \mathbb{R} \\
& \left(x_{1}, \cdots, x_{n}\right) \rightarrow f\left(x_{1}, \cdots, x_{n}\right)
\end{aligned}
$$

The natural inclusion function $[f]$ for $f$ is obtained by replacing each variable $x_{i}$ by an interval $\left[x_{i}\right]$ and each operator or function relating these variables by its interval equivalence as explained in (8) and (9). If each variable $x_{i}$ occurs at most once and all the operators and functions involved in $f$ are continuous, then $[f]$ is minimal. Otherwise, $[f]$ may returns a very pessimistic box containing $f([\mathbf{x}])$ due to the dependency and wrapping effects. In general, natural inclusion functions are not minimal.

Consider the box $[\mathbf{x}]$ as a subset of $\mathbb{R}^{n}$. A union of nonempty and non-overlapping subboxes of $[\mathbf{x}]$ is a subpaving of it. Subpavings are a guaranteed way to approximate a certain compact set of interest $X$. It will be bracketed between an inner subpaving $\underline{X}$ and an outer subpaving $\bar{X}$, as in (13). $X$ contains $\underline{X}$ while $\bar{X}$ contains $X$. We define $\Delta X$ as the subpaving containing the boundary of $X$ between $\underline{X}$ and $\bar{X}$.

$$
\underline{X} \subset X \subset \bar{X}, \text { with } \bar{X}=\underline{X} \cup \Delta X
$$

\section{B. Set Inversion}

Consider a vector function (in bold) $\mathbf{f}: \mathbb{R}^{n} \rightarrow \mathbb{R}^{m}$. Let $Y$ be a subset of $\mathbb{R}^{m}$ and $X$ a subset of $\mathbb{R}^{n}$. We define the vector of variables (in bold as well) $\mathbf{x}=\left(x_{1}, x_{2}, \cdots, x_{n}\right)^{T}$ in $\mathbb{R}^{n}$. The set inversion is the characterization of the reciprocal set $X=\mathbf{f}^{-1}(Y)$ [8] as illustrated in (14):

$$
X=\left\{\mathbf{x} \in \mathbb{R}^{n} \mid \mathbf{f}(\mathbf{x}) \in Y\right\}=\mathbf{f}^{-1}(Y)
$$

Using interval analysis, the recursive algorithm SIVIA (Set Inverter Via Interval Analysis) approximates $X$ as described in (13). It requires an inclusion function $[\mathbf{f}]$ of $\mathbf{f}$ and an initial large box $[\mathbf{x}]$ containing $\bar{X}$. It explores this search domain $[\mathbf{x}]$ by testing its inclusion in $Y$ using $[\mathbf{f}]$ and bisecting it otherwise.

So, if $[\mathbf{f}]([\mathbf{x}]) \cap Y=\emptyset$, then $[\mathbf{x}]$ is guaranteed to not belong to the solution set $X$ and is eliminated. If $[\mathbf{f}]([\mathbf{x}]) \subset Y$, then $[\mathbf{x}]$ is guaranteed to belong to $X$ and is stored in $X$ and $\bar{X}$. If $[\mathbf{f}]([\mathbf{x}]) \cap Y \neq \emptyset$ but, at the same time, $[\mathbf{f}]([\mathbf{x}]) \nsubseteq Y$, we cannot conclude and $[\mathbf{x}]$ is undetermined. If the width of the largest interval component of $[\mathbf{x}]$ is less than a certain positive real number named $\epsilon$, then $[\mathbf{x}]$ is stored in $\bar{X}$. Otherwise, $[\mathbf{x}]$ is bisected into two boxes and the test is applied recursively to each of them. Note that the width of an interval $[x]$ is given by $\omega([x])=\bar{x}-\underline{x}$, while the width of a box $[\mathbf{x}]$ is given by $\omega([\mathbf{x}])$ that returns the width of its largest component. $\epsilon$ represents the precision of the solution approximation. SIVIA is given in the Algorithm. 1.

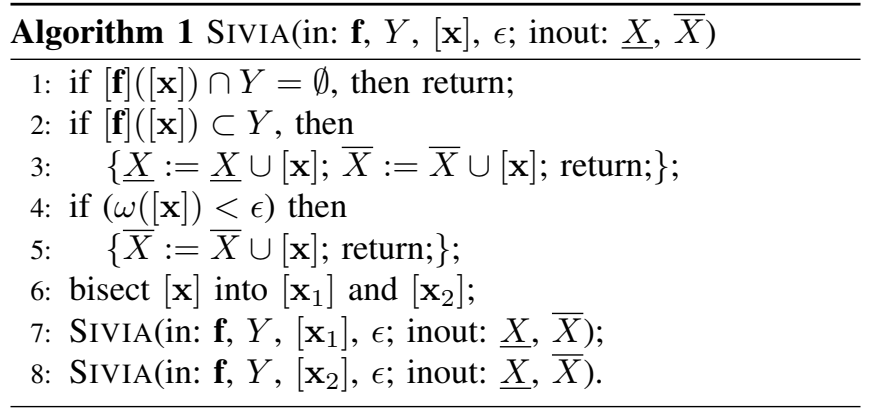

However, if the number of variables is high, bisection increases exponentially the complexity of the computation. In order to resolve this problem and reduce the number of bisections, contractors are used to reduce the box $[\mathbf{x}]$ before bisecting it. A contractor replaces the initial domain $[\mathbf{x}]$ by a smaller one $\left[\mathbf{x}^{\prime}\right]$ who contains as well $X$, i.e. $X \subseteq\left[\mathbf{x}^{\prime}\right] \subseteq[\mathbf{x}]$. In the literature, there exist many techniques to contract boxes as the Gauss elimination procedure, or fixed-point methods. As well, we have the Gauss-Seidel contractor or 
the Krawczyk contractor. The forward-backward contractor, denoted by $C_{\uparrow \downarrow}$, deals with large variable domain $[\mathbf{x}]$. It contracts the domain by isolating each variable separately. The principle of this contractor is given in the following example.

Example 1 Consider the vector of variables $\mathbf{x}=$ $\left(x_{1}, x_{2}, x_{3}\right)^{T}$ linked with the constraint $f\left(x_{1}, x_{2}, x_{3}\right)=$ $x_{1}-x_{2}-x_{3}=0$ and the box $[\mathbf{x}]=[0,4] \times[-1,1] \times[-10,10]$ as the prior domain of the variables. We apply the forwardbackward contraction and we obtain:

$\left[x_{3}\right]=\left[x_{3}\right] \cap\left(\left[x_{1}\right]-\left[x_{2}\right]\right)=[-1,5]$,

$\left[x_{2}\right]=\left[x_{2}\right] \cap\left(\left[x_{1}\right]-\left[x_{3}\right]\right)=[0,4]$,

$\left[x_{1}\right]=\left[x_{1}\right] \cap\left(\left[x_{2}\right]-\left[x_{3}\right]\right)=[-1,1]$.

The new domain is thus $[\mathbf{x}]=[0,4] \times[-1,1] \times[-1,5]$. If we repeat the same procedure with this new domain in order to contract it, it will remain intact. $C_{\uparrow \downarrow}$ comes to a deadlock. Bisecting the search box into two boxes and continuing with the children boxes may solve the problem. We must reunite the children boxes after contraction to get the new $[\mathbf{x}]$.

After adding a contractor, we define the algorithm SIVIAP permitting the characterization of the set $S_{p}$ defined in (15). SiviaP approximates the vector $\mathbf{p}$ such that $\mathbf{g}(\mathbf{p}) \in[\mathbf{y}]$ using the contractor $C_{S_{p}}$. This contractor can be one of the contractors cited below or any combination of them.

$$
S_{p}=\{\mathbf{p} \in[\mathbf{p}] \mid \mathbf{g}(\mathbf{p}) \in[\mathbf{y}]\}=\mathbf{g}^{-1}([\mathbf{y}]) \cap[\mathbf{p}]
$$

SiviAP, given in Algorithm. 2, and SiviA, given in Algorithm. 1, are similar. The main difference is that SIVIAP contracts the boxes before the bisection step.

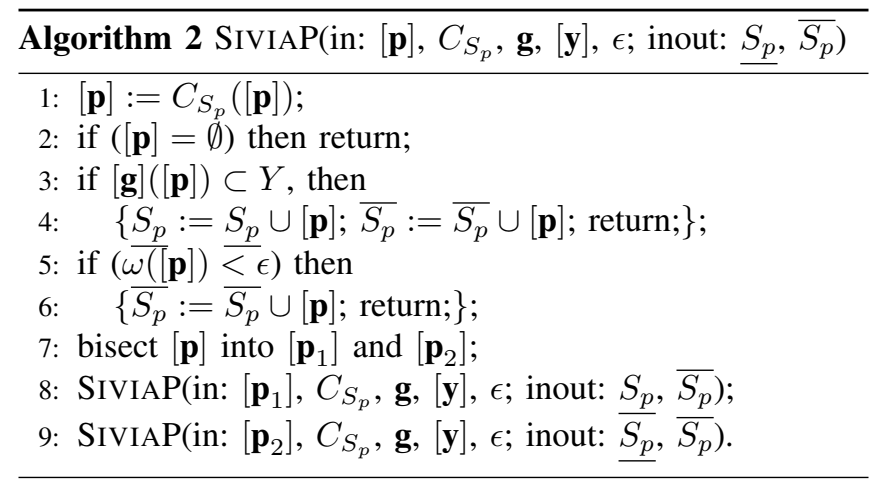

\section{IMPLEMENTATION}

\section{A. Problem Statement}

We consider that the two following elements related to and affecting the vehicle state are known even before the vehicle hits the road:

- The vehicle characteristics such as: the height of its CoG $h$, the wheelbase $l$ as well as $l_{f}$ and $l_{r}$, the tracks $b_{f}$ and $b_{r}$, the total mass $m$, etc.

- The road state modelled by the friction coefficient $\mu$.

As discussed in section II, these elements are related directly through (1) and (3) to the vertical, lateral and longitudinal forces $F_{z}, F_{y}$ and $F_{x}$ of each wheel respectively, and through (2) to the longitudinal and lateral accelerations $a_{x}$ and $a_{y}$ at the CoG. The four vertical forces of the wheels are connected through (4) thus predicting the rollover index. A stable behavior of the vehicle induces a rollover index $L T R$ between -0.8 and 0.8 , a wheel sideslip angle which does not exceed $6^{\circ}$ or does not go under $-6^{\circ}$, and a longitudinal slip ratio $\sigma$ between $-15 \%$ and $15 \%$. These obligations for a stable behavior are discussed in the section II-B and presented in Table. I. It resumes the vehicle stability domain suggested in this paper and gives the three conditions of the system to be stable. The vehicle state can be described by the following state elements $F_{x}, F_{y}, F_{z}, a_{x}$ and $a_{y}$, or a combination of them. Our main purpose is to find, at each instant during the trip, the space of the state vector where the obligations of Table. I are respected and, in consequence, the vehicle is in its stability domain.

The performance of a car can be described by a graph called the "g-g" diagram [17], [9]. It is a plot of the longitudinal versus lateral accelerations at the CoG of a vehicle, scaled with respect to gravity. The linear and nonlinear performance are separated by the adherence circle of a radius equal to $0.4 \mathrm{~g}$. If the accelerations are inside the circle, the vehicle has a linear handling behavior and thus is in a normal driving conditions. Otherwise, its behavior is nonlinear and more risky. Accelerations analysis is very helpful to understand the real situation of the vehicle and handle it eventually.

In consequence, in this paper, we are interested to compute the vector $\mathbf{p}=\left(a_{x}, a_{y}\right)^{T}$ such that $\mathbf{y}=(L T R, \sigma, \alpha)^{T} \in$ $[\mathbf{y}]=[-0.8,0.8] \times[-15,15] \times[-6,6]$ using (1), (2), (3) and (4). The problem to be resolved is the characterization of the set $S_{p}$ presented in (16), where $\mathbf{p}$ is assumed to belong to the large prior search box $[\mathbf{p}]=[-10 g, 10 g] \times[-10 g, 10 g]$. $S_{p}$ is the vehicle stability domain that we search to compute.

$$
S_{p}=\{\mathbf{p} \in[\mathbf{p}] \mid \mathbf{y} \in[\mathbf{y}]\}
$$

An outer approximation $\overline{S_{p}}$ and an inner approximation $S_{p}$ for this $S_{p}$ can be obtained with the desired precision using the algorithm SIVIAP (see the Algorithm. 2) but with some modifications. The new algorithm SIVIAP $\uparrow \downarrow$ is presented in the Algorithm. 3. The main modifications are the following:

- The contractor $C_{S_{p}}$ is chosen to be the forwardbackward contractor $C_{\uparrow \downarrow}$. It is more convenient to use it when dealing with large search domain especially when there is no explicit function $\mathbf{g}$ relating $\mathbf{p}$ to $\mathbf{y}$. In addition, $C_{\uparrow \downarrow}$ will contract the domain $[\mathbf{p}]$, the output variables domain $[\mathbf{y}]$ as well as all the intermediate variables $F_{x i j}, F_{y i j}, F_{z i j}$ for all $i \in\{1,2\}$ and $j \in\{1,2\}$.

- We transmit to $C_{\uparrow \downarrow}$ all the intervals on all the variables $\mathbf{p}, \mathbf{y}, F_{x i j}, F_{y i j}, F_{z i j}$ for all $i \in\{1,2\}$ and $j \in\{1,2\}$. We put them in a single vector as follows: $\mathbf{x}=\left(x_{1}, \cdots, x_{17}\right)^{T}=$ $\left(a_{x}, a_{y}, L T R, \sigma, \alpha, F_{x 11}, F_{x 12}, F_{x 21}, F_{x 22}, F_{y 11}\right.$, $\left.F_{y 12}, F_{y 21}, F_{y 22}, F_{z 11}, F_{z 12}, F_{z 21}, F_{z 22}\right)^{T} \cdot \mathbf{p}$ is a subvector $\mathbf{x}$.

- The initial domain of $\mathbf{x}$ is: $[\mathbf{x}]=[-10 g, 10 g] \times$ $[-10 g, 10 g] \times[-1,1] \times[-100,100] \times[-25,25] \times$ $[-m g, m g] \times \cdots \times[-m g, m g]$. 
- Since we are only interested in $\mathbf{p}$, the algorithm will bisect the box $[\mathbf{x}]$ only along one of the directions $a_{x}$ or $a_{y}$ at step 7 of Algorithm. 3. It bisects along the longest between them. For example, if $[\mathbf{x}]=[-10 g, 10 g] \times[0,10 g] \times[-1,1] \times[-100,100] \times$ $[-25,25] \times[-m g, m g] \times \cdots \times[-m g, m g], \omega\left(\left[a_{x}\right]\right)=$ $20 g>\omega\left(\left[a_{y}\right]\right)=10 g$, then we bisect along $a_{x}$ and we get: $\left[\mathbf{x}_{1}\right]=[-10 g, 0] \times[0,10 g] \times[-1,1] \times[-100,100] \times$ $[-25,25] \times[-m g, m g] \times \cdots \times[-m g, m g]$ and $\left[\mathbf{x}_{2}\right]=$ $[0,10 g] \times[0,10 g] \times[-1,1] \times[-100,100] \times[-25,25] \times$ $[-m g, m g] \times \cdots \times[-m g, m g]$.

- In order to stop the bisection (at step 5), we compare only the widths of the intervals $a_{x}$ and $a_{y}$ with $\epsilon$. Thus, we maintain the expression $\omega([\mathbf{p}])$ which will return the width of the largest interval between them.

With SiviaP $\uparrow \downarrow, \overline{S_{p}}$ and $S_{p}$ are computed at any desired instant during the trajectory. The steering angle $\delta$ is assumed to be known, and it is an input of the algorithm. The main algorithm is summarized in Algorithm 4.
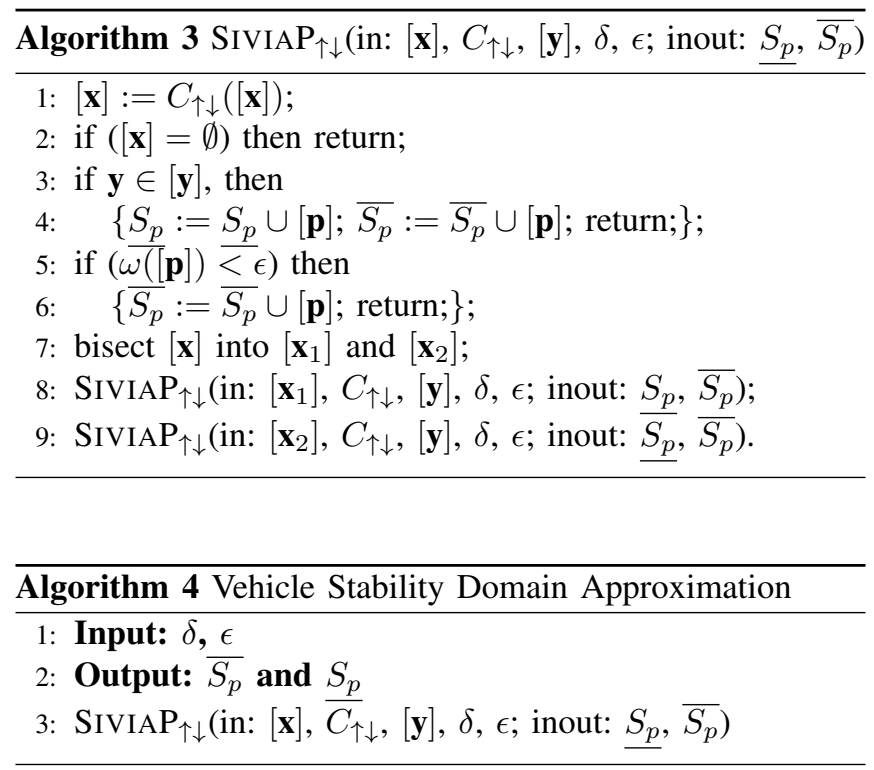

\section{B. Simulation and Results}

In the previous part IV-A, we have presented an interval based method to approximate the vehicle stability domain against three risk factors. In order to validate it, a set of data has been generated using the Callas simulator ${ }^{\circledR}$. Callas is an advanced vehicle dynamics simulator developed by Oktal society (http://www.scanersimulation.com/oktal.html). These data include the trajectory of the vehicle and the corresponding steering angle (see Fig. 5) at a speed of $90 \mathrm{~km} / \mathrm{h}$. The steering angle of the front left tire $\delta_{11}$ and the front right tire $\delta_{12}$ are assumed to be the same. The rear steering angles $\delta_{21}$ and $\delta_{22}$ are assumed to be equal to zero. Then, $\delta=\delta_{11}=\delta_{12}$. The road is dry, thus the friction coefficient $\mu$ is equal to 1 .

Algorithm 4 is applied in three different moments: at the beginning of the trajectory where it is almost a straight line (at the instant $t=0, \delta \simeq 0$ ), in the middle of the first

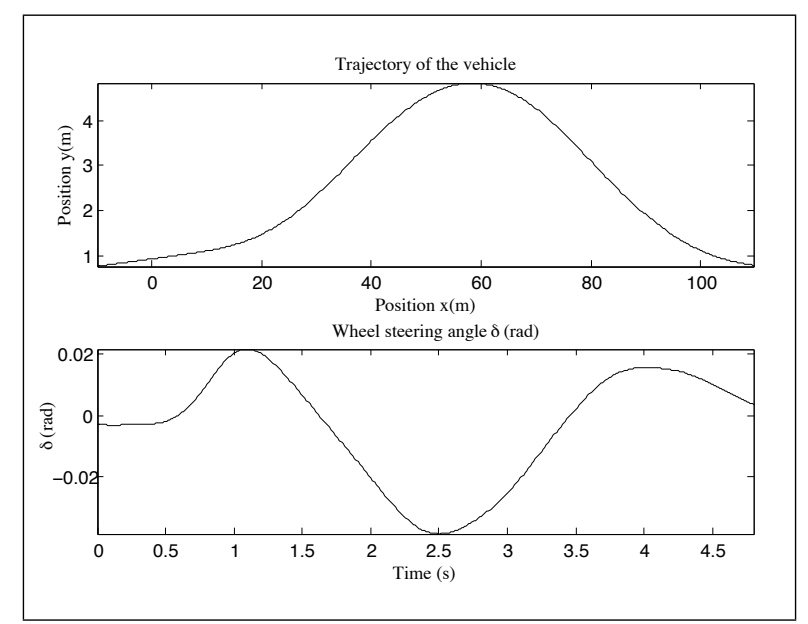

Fig. 5. The trajectory of the vehicle at the speed $90 \mathrm{~km} / \mathrm{s}$ and the corresponding wheel steering angle $\delta(\mathrm{rad})$

cornering $(t=1.2 s, \delta \simeq 0.01 \mathrm{rad})$ and in the middle of the second cornering ( $t=2.5 s, \delta \simeq-0.04 \mathrm{rad}$ ). The second cornering is more severe than the first one. In the three cases, the precision $\epsilon$ is taken equal to 0.4. We use IBEX 2.0, a library for interval arithmetic based on the BIAS/PROFIL library (http://www.emn.fr/z-info/ibex/). Fig. 6, Fig. 7, and Fig. 8 show the result of the Algorithm 4 applied respectively at these three different moments.

These are interval based g-g diagrams. In each figure, the red zone is equivalent to the interior of the adherence circle in the classical $g-g$ diagram (as explained in part IV-A). A main advantage of our interval based g-g diagram is the guaranteed aspect. Thus, with any accelerations couple $\left(a_{x}, a_{y}\right)$ that belongs to one of these red boxes, it is guaranteed that the vehicle has a rollover index $L T R \in$ $[-0.8,0.8]$, a longitudinal slip ratio $\sigma \in[-15,15]$, and wheel side slip angle $\alpha \in[-6,6]$. It is guaranteed that the vehicle is stable againt these three risk factors. The red boxes belongs to the inner subpaving $S_{p}$ which is the vehicle stability domain. On the other hand, in the blue zone, the vehicle is surely unstable. It has at least one unfulfilled condition among the three ones of Table. I. Between the red and the blue zone, there is the boundary where the vehicle state is not guaranteed to be neither stable nor unstable. Its state is undetermined. The yellow boxes represent this boundary denoted by the subpaving $\Delta S$. The outer subpaving $\overline{S_{p}}$ is the union of $S_{p}$ and $\Delta S_{p}$ as explained at the end of the part III-A.

In all the figures, the accelerations of the stability domain (red zone) are still relatively large. This is mainly due to two reasons. The friction coefficient $\mu$, which is equal to one, allows large longitudinal and lateral forces $F_{x}$ and $F_{y}$ respectively in (3) who allow large accelerations as accelerations as well. In a real experiment, this coefficient is lesser than one on a dry road. In addition, in (3), the lateral and longitudinal forces are decoupled. There is no effect of the wheel side slip angle $\alpha$ on the longitudinal force $F_{x}$. As well, the effect of the longitudinal slip ratio $\sigma$ on the 
lateral force $F_{y}$ does not appear in its equation. However, the coupling effect should be taken into consideration as in reality. When $\sigma$ (respectively $\alpha$ ) is added to $F_{y}$ (respectively to $F_{x}$ ), $F_{y}$ (respectively $F_{x}$ ) drops. Thus, the coupling effect limits $F_{x}$ and $F_{y}$ and, in consequence, $a_{x}$ and $a_{y}$.

The approximation of the vehicle stability domain against the three risk factors cited above is given with a precision of 0.4. The total computation cost of the proposed method is about 8 minutes, thus it is not possible to run it on-line for the moment. It remains an off-line method to compute a stability domain which will be used later in the vehicle embedded processing system. A greater precision (a smaller $\epsilon$ ) will reduce the yellow undetermined zone but affect dramatically the computation time.

Now, the question is: how can we use this information? The wheel steering angle can be deduced from the supposed known trajectory. Accelerometers measure the vehicle accelerations. The stability domain is computed off-line on all the trajectory. During the trip, with a simple inclusion test, we can verify whether the measured accelerations couple $\left(a_{x m}, a_{y m}\right)$ belong to the $S_{p}, \Delta S_{p}$ or none of them. According to the result, the appropriate action will be taken. For example, if $\left(a_{x m}, a_{y m}\right)$ is in a red box, thus the vehicle behavior is linear, and there is no rollover danger. The vehicle may continue in its actual state. However, if $\left(a_{x m}, a_{y m}\right)$ is in a red box but near the boundary, we should act such as the accelerations are drown back to the nearest red box toward the center of the red zone, either by a breaking or any other command. If $\left(a_{x m}, a_{y m}\right)$ does not belong to $\overline{S_{p}}$ ( $=S_{p} \cup \Delta S_{p}$ ), thus, it belongs to the blue zone of instability.

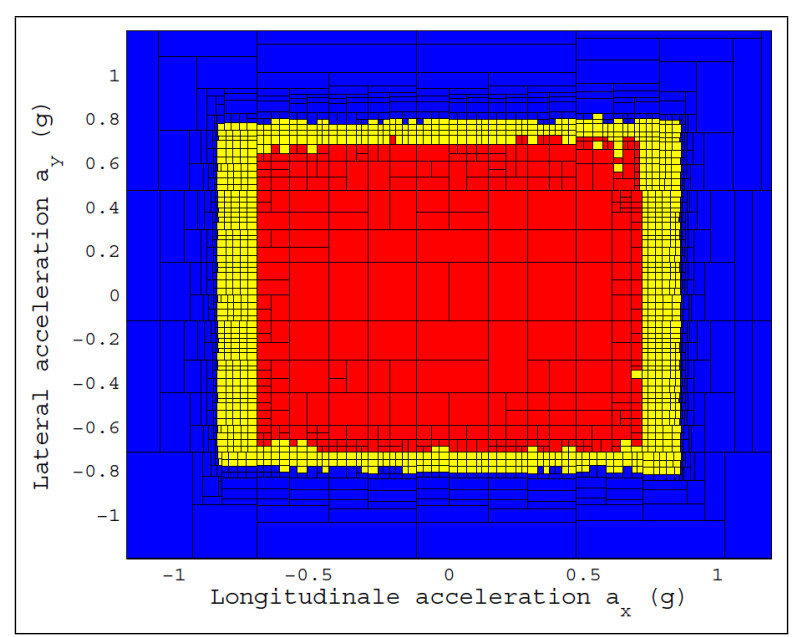

Fig. 6. Longitudinal and lateral accelerations $a_{x}$ and $a_{y}$ in $g$ units at the beginning of the trajectory $(\delta=0 \mathrm{rad})$

\section{CONCLUSIONS}

In this paper, we have presented a new approach to compute a stability domain of the vehicle using interval analysis. We have computed subpavings of the accelerations $a_{x}$ and $a_{y}$ that correspond to a stable vehicle state by observing the three main elements: the rollover index,

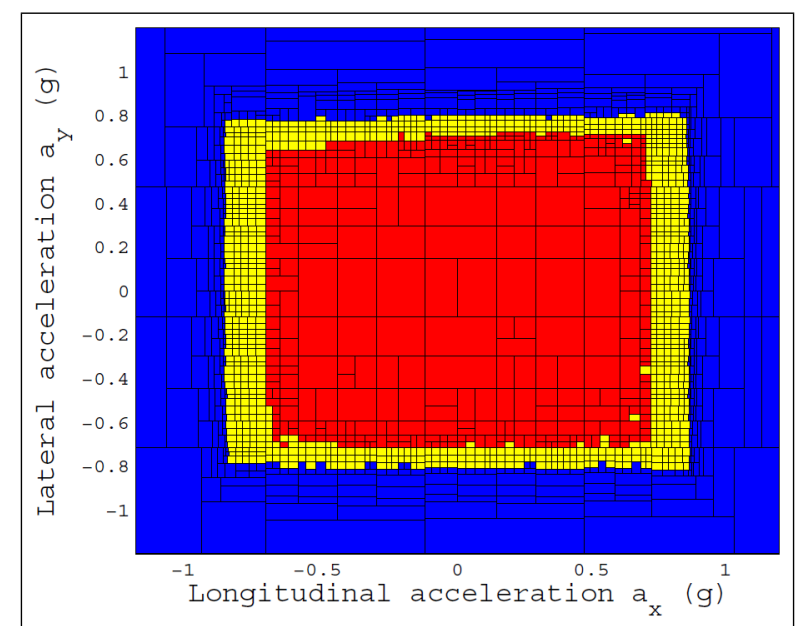

Fig. 7. Longitudinal and lateral accelerations $a_{x}$ and $a_{y}$ in $\mathrm{g}$ units at the first cornering $(\delta=0.01 \mathrm{rad})$

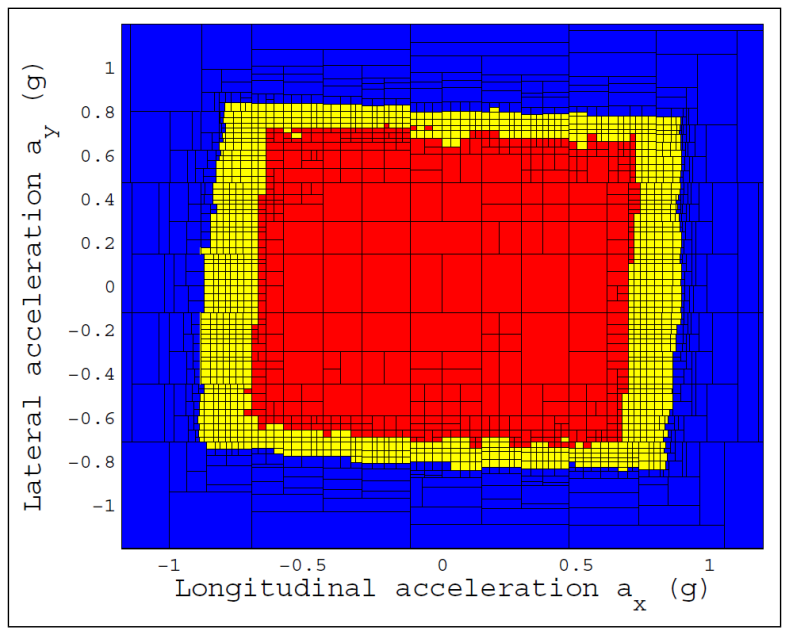

Fig. 8. Longitudinal and lateral accelerations $a_{x}$ and $a_{y}$ in $\mathrm{g}$ units at the second cornering $(\delta=-0.04 \mathrm{rad})$

the longitudinal slip ratio and the wheel side slip angle. Alongside the state estimation and risks anticipation, this method gives a limit of the vehicle behavior. Below this limit, the stability is guaranteed. Above it, a dangerous behavior may be imminent.

In future works, we intend to compute, on the whole trajectory, the vehicle velocities $V_{x}$ and $V_{y}$ that correspond to a small "stable" slip angle $\beta$ at the vehicle center of gravity in addition to the accelerations. Optimization of the algorithms is intended in order to compute these velocities in real-time.

\section{REFERENCES}

[1] S. Antonov, A. Fehn, and A. Kugi, Unscented Kalman filter for vehicle state estimation, IEEE International Conference on Robotics and Automation, Vehicle System Dynamics, vol. 49, no. 9, pp.14971520,2011

[2] N. Bouton, R. Lenain, B. Thuilot, J.-C. Fauroux, A rollover indicator based on the prediction of the load transfer in presence of sliding: application to an all Terrain Vehicle, IEEE International Conference on Robotics and Automation, pp.1158-1163, Roma, 2007.

[3] H. Dandach, F. Abdallah, J. De Miras et A. Charara, Vehicle Dynamics Estimation using Box Particle Filter, The 12th International Conference on Control, Automation, Robotics and Vision (ICARCV), Guangzhou, 2012. 
[4] M. Doumiati, A. Victorino, D. Lechner, G. Baffet, and A. Charara, Observers for vehicle tyre/road forces estimation: experimental validation, Vehicle System Dynamics, vol. 11, no. 4, pp. 1345-1378, Oct. 2010.

[5] V. Drevelle, and P. Bonnifait, iGPS: Global Positioning in Urban Canyons with Road Surface Maps, IEEE Intelligent Transportation Systems Magazine, vol. 4, no. 3, pp. 6-18, 2012.

[6] R. Ghandour, A. Victorino, A. Charara, and D. Lechner, A vehicle skid indicator based on maximum friction estimation, 18th International Federation of Automatic Control Conference (IFAC) World Congress, Milano, 2011.

[7] R. Ghandour, F. H. R. Da Cunha, A. Victorino, A. Charara, and D. Lechner, A method of vehicle dynamics prediction to anticipate the risk of future accidents: Experimental validation, 14th International IEEE Conference on Intelligent Transportation Systems (ITSC), Washington, DC, 2011.

[8] L. Jaulin, M.Kieffer, O. Didrit, and E. Walter, Applied Interval Analysis, Springer, Great Britain, 2001.

[9] R. N. Jazar, Vehicle Dynamics: Theory and applications, Springer, 2008.

[10] U. Kiencke and L. Nielsen, Automotive Control Systems for Engine, Driveline, and Vehicle, Springer-Verlag, Germany, 2000.

[11] L. Menhour, D. Lechner, A. Charara, Vehicle steering control based on robust control for high lateral accelerations: Experimental evaluation, 13th International IEEE Conference on Intelligent Transportation Systems (ITSC), Madeira Island, 2010.

[12] R.E. Moore, Interval Analysis, Prentice-Hall, Englewood Cliffs N. J., 1966.

[13] J. Moras; V. Cherfaoui, and P. Bonnifait, Credibilist occupancy grids for vehicle perception in dynamic environments, IEEE International Conference on Robotics and Automation, Shanghai 2011.

[14] H.B. Pacejka, Tyre and Vehicle Dynamics, Butterworth-Heinemann, Oxford, 2002.

[15] G. Phanomchoeng and R. Rajamani, Real-Time Estimation of Rollover Index for Tripped Rollovers with a Novel Unknown Inputs Nonlinear Observer, American Control Conference (ACC), Montréal, 2012.

[16] A. Petrovskaya and S. Thrun, Model Based Vehicle Tracking in urban environments, IEEE International Conference on Robotics and Automation, Kobe 2009.

[17] R. Rajamani, Vehicle Dynamics and Control, Springer US, 2006. 